\section{Weitere Mittheilungen über einen Fall von chronischem Hydrocephalus bei hereditärer Syphilis.")}

Von Dr. Julius Heller in Charlottenburg-Berlin.

Schon häufig ist auf den Missstand hingewiesen worden, dass wichtige, nicht letal endende Krankheitsfalle in einem gewissen, mehr zufälligen Zeitpunkte veröffentlicht werden, ohne dass weitere Mittheilungen über den späteren Krankheitsverlauf in die Oeffentlichkeit gelangen. Von diesem Gesichtspunkte aus möchte ich uiber einen Fall berichten, den ich boreits im Jahre 1892 in der Deutschen medicinischen Wochenschrift No. 26 unter dem Titel: „Ein Fall von chronischem Hydrocephalus bei hereditärer Syphilis" veröffentlicht habe.

Ich gebe noch einmal ein Referat der Krankengeschichte. Im Juni 1890 legte ich bei der 36 Jahre alten Primipara H. wegen Wehenschwäche den Forceps an. Der Umstand, dass bei der alten Erstgebärenden kein Dammriss entstand, beweist, dass der Kopf des Kindes jedenfalls nicht pathologisch vergrössert war. Da der Knabe bei künstlicher Ernährung nicht gedieh, wurde eine Amme genommen, die von mir genau untersucht wurde. Nach vorübergehender Besserung verschlechterte sich der Zustand des Kindes; es traten deutliche Zeichen von Enteritis und Atrophie auf.

Ende Juli zeigte sich ein zweifellos syphilitisches Exanthem, das bei specifischer Therapie (Sublimatbäder, Calomel 0,01 dreimal täglich) schwand. Gleichzeitig besserte sich das Allgemeinbefinden (Ernährung nach wie vor durch die von der Art der Erkrankung des Kindes in Kenntniss gesetzte Anrme), und Anfang October konnte der Knabe als körperlich gesund und geistig seinem Alter entsprechend entwickelt betrachtet werden. Ende Januar $1891 \mathrm{sah}$ ich das Kind wieder. Es bot vollig das Bild eines Hydrocephalus chronicus dar. Der Kopf war unförmig gross, das Gesicht dagegen anffallend klein, die Stirn ungewöhnlich hoch, die Scheitelbeine nach aussen gebogen, balkonartig hervortretend. Die grosse Fontanelle war männerbandgross, die Stiinbeinfontanelle deutlich festzustellen. Die früher normale Behaarung des Kopfes inclusive Augenbrauen und Augenwimpern fehlte völlig; die kahle Kopthaut war von dicken, verzweigten, blau durchschimmernden Venen durchzogen. Die Messung des Umfanges des Schädels um Kinn und Scheitel und um die Stirn in der Höhe der Tubera frontalia sowie die Bestimmung der librigen Durchmesser ergab eine beträchtliche Vergrösserung gegen die Norm. (Die genauen Maassangaben sind in der Originalarbeit angegeben.) Krämpfe wurden nicht constatirt. Der geistige Zustand des Kindes war weit unter der Norm.

Bei antisyphilitischer Therapie (Jodkali $1-2: 120$, dreimal täglich ein Kindcrloffel, im ganzen ctwa $20 \mathrm{~g}$ Jodkali) trat Ruckgang der geschilderten Erscheinungen und nach fünf Monaten Heilung ein. Der Knabe konnte Juni 1891 als körperlich und geistig normal betrachtet werden.

In nieiner damaligen Publication habe ich ausführlich nachgewiesen, dass in der That Hydrocephalus und Syphilis vorlag, dass Rachitis auszuschliessen war. Die Syphilis musste allein aus den Symptomen diagnosticirt werden, die Anamnese liess im Stich, da ich den Vater des (unehelichen) Kindes, eine in Berlin bekannte, jetzt verstorbene Persönlichkeit, nicht kennen lernte.

Wenn auch mein der Privatpraxis entstammender und daher dauernd beobachteter Fall als so wichtig für die Frage der syphilitischen Aetiologie des Hydrocephalus betrachtet wurde, dass er in vielen neuen Lehrbüchern (Henoch, Heubner, Oppenheim, Joseph) citirt wurde, so waren doch noch zwei Einwände denkbar. Es konnte gefragt werden: 1. Liegt nicht etwa eine Verwechselung eines rachitischon mit einem hydrocephalen Schädel vor? 2. Handelt es sich bei dem Kinde wirklich um hereditäre Syphilis? Sind so schwere Gefässveränderungen, wie sie beim syphilitischen Hydrocephalus supponirt werden muissen, annehmbar, wenn als einziges Zeichen der bereditären Syphilis ein relativ leichtes Exanthem vorangegangen ist?

Auf beide Fragen hat die Zeit eine, icli mochte sagen, stringente Antwort gegeben.

Ich habe das gesundheitliche Schicksal des Knaben dauernd verfolgen können, ich habe ihn fast in jedem Jahre mehrere Male gesehen, ich bin über alle nicht von mir behandelten Krankheiten des Kindes genau informirt worden. Der jetzt $7 \%$ Jahre alte Knabe hat nie ein Symptom von Rachitis gehabt. Zahn- und Knochenentwickelung war durchaus normal. In seinem dritten und vierten Lebensjahre konnte man den Knaben als gross und kräftig und als sehr gut gewachsen bezeichnen. $\mathrm{Nie}$ hat er einen jener, bei rachitischen Kindern so bäufigen Zufälle (Convulsionen, Spasmus glottidis u. s. w.) gehabt. Man darf wohl als sicher annehmen, dass eine schwere Rachitis, die sogar zu hydrocephaloiden Schädelveränderungen führt, sich auch sonst noch manifestirt haben würde.

1) Nach einer Demonstration im Verein für innere Medicin am 6. December 1897.
Bis zur Vollendung des siebenten Lebensjahres hat auch die hereditäre Syphilis keine weitere Erscheinungen gemacht. Es lag demnach auch keine Veranlassung vor, die antisyphilitische Cur prophylaktisch zu wiederholen. Im Śmmer 1897 erkrankte der Knabe an einer doppclseitigen Augenentzündung, die von dem Augenarzt Herrn Dr. Wertheim als interstitielle Keratitis diagnosticirt und dementsprechend mit Atropin und Schmiercur behandelt wurde. Die Schmiercur wurde bald wicder ausgesetzt. Im August wurde mir der Knahe wegen eines beginnenden, das Allgemeinbefinden sehr beeintrïchtigenden Knochenleidens wieder zugeführt. Ich nahm folgenden Status auf.

Die Haut des Gesichts (vor allen die sichtbaren Schleimhäute) des für sein Alter grossen, gut gebauten Knaben ist anämisch. Die Pupillen ad maximum dilatirt (Atropin), Bei auffallendem Lichte deutlich beiderseits an den tiefer liegenden Trübungen interstitielle Keratitis zu erkennen. Spiegelung des Augenhintergrundes unmöglich. Die Functionen des Körpers sind normal, nur ist der Appetit etwas schwach. Die Reflexe, insbesondere der Kniereflex, durchaus unverändert. Am Gehörorgan keine Anomalie. An den oberen Schneidezähnen (II. Dontition) sind deutliche Hutchinson'sche Veränderungen festzustellen. An der linken Hälfte der Unterlippe findet sich ein cinem Condylom ähnliches, speckig belegtes Gebilde. An 'der linken Tibia, unterbalb der Tuberositas, besteht eine etwa $4 \mathrm{~cm}$ lange, $2^{1 / 2} \mathrm{~cm}$ breite, zweifellos vom Periost ausgehende Geschwulst. (Periostales Gummi.)

Genauer beschreiben will ich die am linken Humerus sitzende Geschwulst. Auch diese war ein periostales Gummi. Die Geschwulst lag gerade nach vorn, wenn der Knabe die herabhängende Handfläche gegen die Extremität legte, etwa $1 \mathrm{~cm}$ oberhalb der Epiphysengrenze. Der Tumor war, nit dem Tasterzirkel gemessen, $4^{3} / 4 \mathrm{~cm}$ in Längen- und Breitenausdehnung gross. Der Unffang des Armes über der Geschwulst war $18 \% / 2 \mathrm{~cm}$ (rechts $15 \mathrm{~cm}$ ). Die Haut über dem Tumor war durchaus normal, der Tumor selbst ging, ohne Verbindung mit der Haut zu haben, direkt vom Knochen aus.

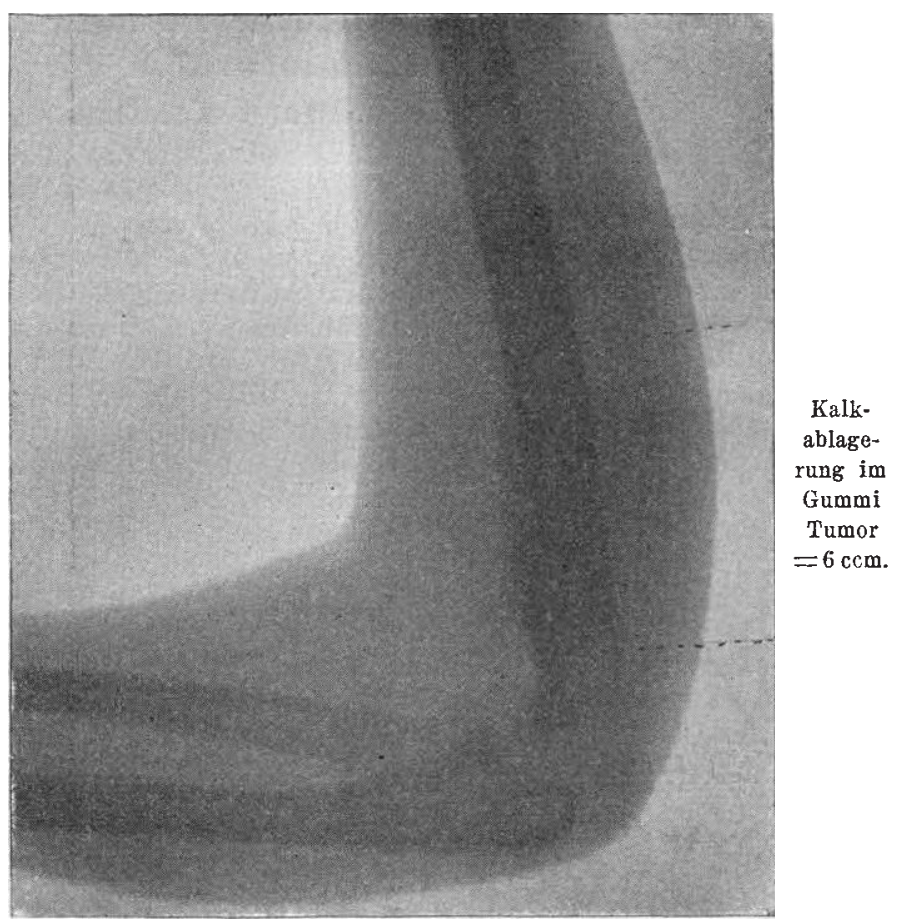

Die Therapie bestand in Ruhigstellung der Gelenke, lokaler Anwendung von Hydrargyrumparaplast, interner Darreichung von Jodkali (4-6:200, dreimal täglich ein Kinderlöffel). Nachdem der Knabe acht Flaschen Jodkali verbraucht hatte (im ganzen circa $40 \mathrm{~g}$ Jodkali), wurde Jodeisensyrup gegeben. Grosse Sorgfalt wurde der Ernährung zugewendet. Die Therapie hatte den erhofften Erfolg, der Knabe wurde völlig geheilt.

Ich habe nun von dem periostalen Gummi auf der Hohe seiner Entwickelung eine Röntgen-Photographie durch Herrn A. W. Hirschmann machen lassen. Das Skiagramm hat ein bemerkenswerthes Resultat gegeben. Die Aufnahme geschah in der Weise, dass der Ellenbogen des Kindes auf der Platte fixirt und die Röhre etwa in $25 \mathrm{~cm}$ Entfernung uher der Geschwulst aufgestellt wurde. Man sieht nun im Skiagramm an der dem Tumor entsprechenden Stelle einen $6 \mathrm{~cm}$ langen, kreissegmentförmig gestalteten Schatten der deutlich erhaltenen Contour des Humerusschattens auf der Innenseite des letzteren aufliegen. Dieser aufliegende Schatten ist an seiner breitesten Stelle $4-5 \mathrm{~mm}$ breit, die Intensität des Schattens ist in den einzelnen Abschnitten eine ganz verschiedene.

Wie ist dieser Schatten zu erklären? Eine so wenig blutreiche, kalklose Geschwulst wie ein Gummi kommt im Röntgenbilde nicht zur Darstellung. Wenn sie doch zur Darstellung gelangt wäre, so hätte ihre 
Forn eine andere sein mussen. Eine ostale Veränderung, eine Exostosenbildung '), eine Auftreibung des Knochens kann nicht vorliegen. Abgesehen davon, dass der klinische Verlauf, der schuelle Schwund des Tumors gegen eine derartige Knochenveränderung spricht, ist im Schattenbilde die normale Contour des Humerus deutlich von der Auflagerung abzugrenzen. Nur eine Auffassung erklärt ungezwungen die eigenartige Schattenbildung: sie ist der Ausdruck der Kalkablagerung an der Grenze des Knochens und des periostalen Gummis.

Es ist bekannt, dass bei jedem periostalen Gummi Auflösung der Kalksalze an der Knochengrenze stattfindet. Der pathologische Ausdruck für diesen Vorgang ist die Bildung der sogenannten Knochennarbe. Ein Theil der aufgelösten Kalksalze wird ror ihrer Resorption im Gummi gewissermaassen abgelagert. So hat aber das Röntgenbild einen gewissermaassen pathologisch-anatomischen Vorgang mit ausserordentlicher Scharfe zur Anschaung gebracht. ${ }^{2}$ )

Ich halte diesen Befund für interessant. Bisher hat die Rontgenphotographie für die syphilitischen Knochenveränderungen keine besonders hervortretenden Resultate ergeben. Ohne Erfolg hat Herr Prof. Buka 1896 für mich periostitische Knochenveränderungen bei einem Kranken der Syphilisklinik der Charite (Geh. Rath Lewin) röntgographirt. Bei einem Fall säbelscheidenförmiger Verkı ummung der Beine infolge von Lues hereditaria tarda (Syphilisklinik der Charite von Prof. Lesser), der von Herrn Stabsarzt Dr. Bieck in der Gesellschaft der Chariteärzte vorgestellt wurde, ergab die Röntgenphotographie wohl die eigenartige Krümmung, aber keine specifischen Veränderungen wieder.

Der interessante Befund des Skiagramms kann die ohnehin unzweifelhaft feststehende Diagnose periostales Gummi nur bestätigen. Es dürfte aber damit der Beweis erbracht sein, dass der in seinem ersten Lebensjahre an einem chronischen Hydrocephalus erkrankte Knabe in der That an einer schweren ererbten Syphilis litt.

Die 71/2 Jahre hindurch fortgesetzte Beobachtung des einen Falles machte ihn meines Erachtens für den ätiologischen Zusammenhang $\mathrm{z}$ wischen Hydrocephalus und Syphilis wichtig. Trotzdem in den $51 / 2$ Jahren, seitdem meine erste Publication erschienen ist, einige weitere Fälle von Hydrocephalus bei hereditärer Lues publicirt sind (vgl. die Arbeit von Elsner, Jahrbuch für Kinderheilkunde 1896), ist ein weiterer Fall von Heilung mir nicht bekannt geworden. 